\title{
EL MODELADO y SIMULACIóN PARA LA COMPRENSIóN DE LOS SISTEMAS DE PRODUCCIóN DE POZOS PETROLEROS
}

\author{
Eliécer Pineda Ballesteros, ${ }^{71}$ fernando Enrique Calvete González, ${ }^{72}$ \\ fernando Ruiz Díaz ${ }^{73}$
}

\section{RESUMEN}

Este artículo muestra los resultados de una propuesta de investigación acerca de si el uso del modelado y la simulación pueden contribuir en la comprensión de los fenómenos físicos asociados a la producción de pozos petroleros.

La investigación comprende la modelación del fenómeno incluyendo al yacimiento y el pozo. Se toma como referente teórico la Ley de Darcy y una vez construidos los modelos del fenómeno se reconstruye un micromundo que facilita la experimentación y la construcción de modelos. La parte final del proceso contempla el uso del micromundo en sesiones de trabajo con los estudiantes, y la posterior validación de los efectos de su uso comparando su desempeño con el de aquellos que no hicieron parte de las sesiones de trabajo.

Si bien es cierto que la muestra no fue significativamente grande, si llama la atención el hecho de que los estudiantes que concurrieron al seminario hayan tenido un mejor desempeño respecto a los que no asistieron, al comparar las calificaciones definitivas de la asignatura sistemas de producción.

Palabras clave: dinámica de sistemas, simulación, micromundos, ley de Darcy, sistemas de producción de pozos petroleros.

\section{ABSTRACT}

This article shows the results of a research proposal regarding if the use of models and simulations can contribute to the comprehension of the physical phenomenon associated with the production of oil wells.

The research entails the modeling of the phenomena, including the oilfield and well. The Darcy Law is taken a theoretical reference, and once the models of the phenomena are built, a microworld is reconstructed that facilitates the experimentation and the construction of models. The final area of the process contemplates the use of the micro-world in work sessions with the students. This final part also includes the subsequent validations of the use of these micro-

71 Ingeniero de Sistemas y Economista, M. Sc. Docente, Universidad Nacional Abierta y a Distancia. <eliecer. pineda@unad.edu.co>

72 Ingeniero de Petróleos, M. Sc. Profesor Asistente, Universidad Industrial de Santander. <fcalvete@uis.edu.co>

73 Ingeniero de Sistemas, M. Sc. Profesor Asociado, Universidad Industrial de Santander. <fruizd@uis.edu.co> 
worlds, contrasting the performance of the students that used them, with that of whom did not take part of the work sessions.

Even though it is true to say that the sample was not meaningfully big, the fact that the students that went to the seminar had a better performance in comparison with those students who did not participate in it, is something that draws one's attention, specially when contrasting the final grades in the production systems class.

Key words: Systems dynamic, simulation, micro-worlds, Darcy's Law, production systems of oil wells.

Recibido: 11 de marzo de 2009

Aceptado: 3 de abril de 2009

\section{INTRODUCCIóN}

En la formación de los ingenieros en Colombia, específicamente en lo que se denomina «el ciclo básico» o los primeros dos años de formación, la gran mayoría de las asignaturas corresponden a exposiciones teóricas de modelos matemáticos que, por lo general, son realizadas por parte del docente. Posteriormente el estudiante deberá memorizarlos y ganar experticia en su manipulación para entonces hallar soluciones a problemas planteados por el docente. Como resultado de esta actividad quedan dudas si efectivamente el estudiante estaría en capacidad de dar cuenta del fenómeno estudiado o si solo está capacitado para manipular eficazmente tales modelos.

La hipótesis que guía la investigación desarrollada por Pineda (2007) sugiere que los estudiantes cuando hacen uso de los modelos dinámicos, mediante su simulación, ganan en la comprensión de lo modelado. Debe entenderse que el uso de modelos como estrategia de aprendizaje no implica necesariamente la construcción de los mismos, cosa que sería deseable, sino que se refiere a una capacidad para leer los modelos usando los lenguajes en que fueron construidos, dentro de los cuales se encuentra el lenguaje de las matemáticas.

Autores del área de dinámica de sistemas plantean ciertas ideas que permiten apoyar la anterior hipótesis, entre ellos Radzicki et al. (2002) quienes proponen algunos argumentos para que los estudiantes de ingeniería aprendan dinámica de sistemas, Estos son:

- Podrían ver la naturaleza genérica de los flujos y niveles y las estructuras de realimentación.

- Tendrían una herramienta interdisciplinaria que puede ser usada para hallar soluciones a los sistemas dinámicos que son generalmente percibidos como estáticos.

- Desarrollarían habilidades para resolver e integrar problemas multidisciplinarios.

- Aprenderían, vía procesos experimentales, desarrollando competencias esenciales y procesos de aprendizaje centrados en el aprendiz.

- Les aportaría una herramienta para estudiar la interacción entre tecnología y sociedad. 
Es en este contexto donde la investigación buscó medir qué tanto influye, en la capacidad de comprender un fenómeno, el que un estudiante trabaje con micromundos realizando experimentos guiados por el docente.

\section{MARCO REfERENCIAL}

\section{Producción de pozos petroleros}

En el ámbito de la ingeniería de petróleos y, en especial, el ingeniero de producción debe desarrollar las competencias que le permitan hacer producir los pozos de aceite y para ello se requiere que comprenda los principios que rigen tanto el movimiento del aceite como el del gas y el agua desde la formación hasta el cabezal del pozo. Según Nind (1987) las principales fases que dan cuenta del comportamiento del pozo son las pérdidas de presión en un flujo vertical bifásico, el comportamiento de la relación gas-aceite y los problemas de bombeo de los pozos profundos que definen los índices de productividad. Así mismo indica que el ingeniero ha de tener las competencias que le permitan hacer uso del arsenal teórico pero con plena conciencia de lo que está haciendo. También hace notar que un pozo productor de aceite o petróleo es solo una parte de un sistema complejo el cual comprende el yacimiento, los pozos mismos y las instalaciones superficiales.

\section{Pensamiento sistémico y dinámica de sistemas}

El pensamiento sistémico aporta elementos conceptuales que permiten ver el mundo como si este fuera un sistema. La trampa que debe evitarse a toda costa es la de creer que los sistemas tienen existencia física; debe quedar claro que un sistema es una idea que se percibe al observar conjuntos de partes que están interrelacionadas y que en conjunto tienen un fin o propósito y además dichas partes están regidas por relaciones de jerarquía.

De otra parte y emparentada con el pensamiento sistémico emerge en los años cincuenta la dinámica de sistemas, creada por el profesor J. W. Forrester del MIT, como una forma de modelado que permite representar la dinámica propia de un fenómeno a partir de la definición de la estructura que a este le subyace. En este trabajo la dinámica de sistemas se concibe como una unidad paradigma-lenguaje, pues a medida que se representa un fenómeno se va adquiriendo mayor destreza para identificar los elementos, las relaciones y los bucles de realimentación. Los modelos construidos con dinámica de sistemas utilizan los cinco lenguajes de formalización descritos ampliamente por Andrade et al. (2001).

\section{La simulación y su relación con la pedagogía}

De la relación que se puede establecer entre la simulación y el aprendizaje comienza a aparecer una serie de cuestionamientos alrededor de lo que el sistema educativo tradicional propone y hace. Al respecto De Zubiría (2006) dice que «la escuela tradicional abandonó el pensamiento y concentró sus esfuerzos en los aprendizajes mecánicos y particulares obtenidos mediante la reiteración de la exposición y la práctica». Esta situación se refleja principalmente en la dificultad que 
los estudiantes tienen a la hora de realizar acciones como la redacción de un informe y aún más cuando se trata de escribir un ensayo.

Una de las principales prácticas de la escuela tradicional consiste en enseñar las ciencias desprendiéndolas de su carácter abstracto y explicativo de la realidad, haciendo que el estudiante pierda la posibilidad de cualificar su representación del mundo y de desarrollar su pensamiento, manteniendo así las representaciones que ha elaborado en su niñez, y debilitando por consiguiente su natural tendencia a preguntarse acerca de lo que ve y no comprende.

En una experiencia relatada por Marchisio, et al. (2004) se describe el uso de los simuladores para el aprendizaje de la mecánica cuántica, cronológicamente posterior al estudio de la mecánica clásica. En el texto se sostiene que, específicamente, puede destacarse como aspecto relevante la necesidad de un cambio sustancial de paradigma e indica además que su objeto de estudio, para el caso de la mecánica clásica, no es accesible a nuestros sentidos en forma directa.

\section{MODELOS y MICROMUNDO}

\section{Los modelos}

Para el propósito de la investigación se realizaron tres modelos introductorios a la simulación dinámica, el modelo del crecimiento poblacional, el modelo del CDT y el modelo presa predador. El cuarto modelo corresponde a un prototipo de la evolución de la representación del fenómeno de producción de pozos petroleros.

\section{Descripción del primer prototipo}

Para su descripción se toma como referencia a Zora (2008). Basado en sus trabajos sobre la mecánica de flujos, el ingeniero francés Henry Darcy (1803-1858) descubrió que existe una relación entre:

- la cantidad de agua («Q», unidad: [m/s]) que fluye a través de una superficie

- la superficie («A», unidad: [m2])

- el gradiente hidráulico («i», determinado por la distancia «l» recorrido y la diferencia «h» de la altura del nivel freático), y

- el coeficiente de permeabilidad kf (un coeficiente específico para cada tipo de roca

Como resumen de sus trabajos se puede expresar la «ley de DARCY» en la forma:

$\mathrm{kf}=\mathrm{Q} /(\mathrm{i}$ x A) (unidad para kf: [m/s]). El kf describe la resistencia hidráulica de una roca transcurrida por el agua y permite determinar su permeabilidad. El valor del $\mathrm{kf}$ depende de las propiedades del agua (temperatura, peso específico, viscosidad) y del acuífero (poros, grietas). 


\section{Diagrama de flujos y niveles}

El diagrama de flujos y niveles es una descripción en términos de razones de cambio y acumulaciones que en el lenguaje de la dinámica de sistemas se representa con grifos y recipientes, es en este último en el que se acumula el cambio. En el siguiente gráfico se observa el modelo de la ley de Darcy.

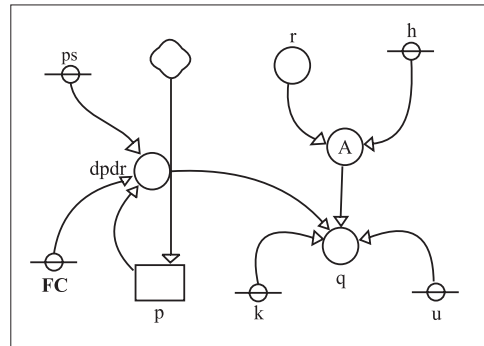

figura 1. Diagrama de flujos y niveles de la ley de Darcy

En el nivel P se acumula la variación de la presión que está relacionada con un valor límite PS o presión estática del yacimiento y la presión en el wellbore o pwf. El caudal q se define a partir de la relación existente entre el cambio de la presión $d p d r$ y otros factores como el radio de drenaje $\mathrm{r}$, el horizonte de producción h, la viscosidad u y la permeabilidad k. Teóricamente la ley de Darcy se expresa como sigue:

$$
\frac{Q}{A}=\frac{k}{\mu} \frac{d p}{d r}
$$

\section{Comportamiento}

El siguiente gráfico muestra cómo evoluciona la presión, a medida que lo hace el radio de drenaje. Si bien es cierto esta es una curva ideal, se aproxima bastante al comportamiento que normalmente expresan estas variables en la realidad.

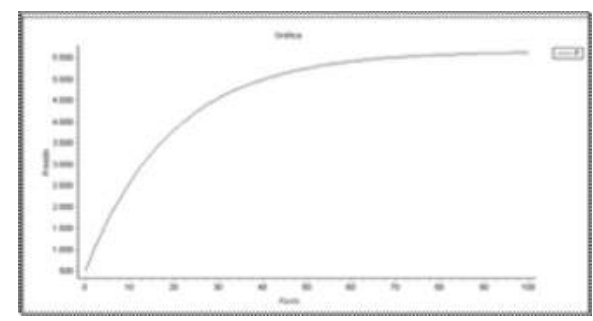

figura 2. Comportamiento del modelo

La importancia de las gráficas radica en que el estudiante podrá ver cómo va cambiando una variable respecto de la otra, lo cual favorece que las explicaciones se den desde una perspectiva dinámica y no una estática con todo lo que eso implica. 


\section{Micromundo}

Para el trabajo con los modelos se propuso retomar un micromundo realizado por Lizcano A. et al. (2002) en una investigación previa, sobre el uso de los modelos en economía realizando las adaptaciones correspondientes. El micromundo está conformado por dos grandes componentes, el de Administración y el Micromundo; en este último se realiza el ejercicio central de presentación e interacción con los contenidos del software, a partir del cual se busca el aprendizaje del estudiante, mediante la experimentación simulada.

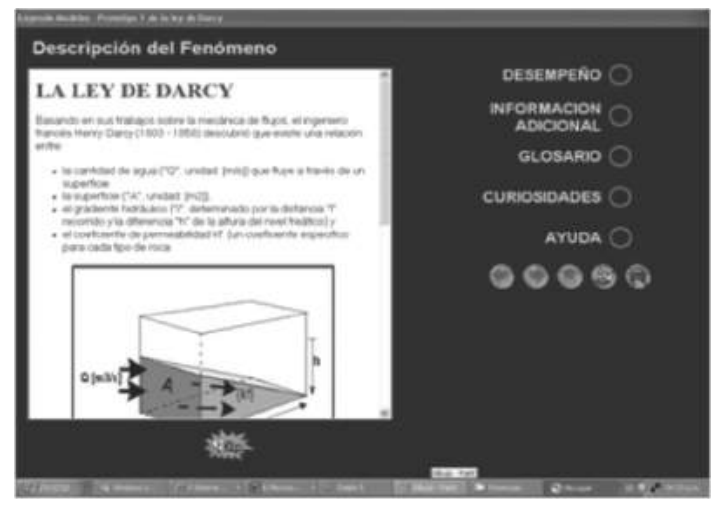

figura 3. Ambiente Leyendo Modelos - Pantalla de Descripción del fenómeno

El núcleo del Micromundo está conformado por dos módulos, cada uno de los cuales está caracterizado porque desarrolla un conjunto de modelos. El introductorio a la dinámica de sistemas inicia a los estudiantes en la utilización de los lenguajes dinámico-sistémicos a través de tres modelos que recrean situaciones bien conocidas por ellos como el caso del crecimiento de una población, el CDT y un modelo de presa predador; y en el módulo de presentación de los prototipos se tiene acceso al modelo que representa la temática de la ley de Darcy. En él se presenta la posibilidad de modificar el modelo propuesto y, además, permite la construcción de otros modelos al facilitar la comunicación con un software de modelado; una vez construido el modelo, éste se puede importar al micromundo en el cual se podrán realizar las experimentaciones de acuerdo a como se programen por el docente.

\section{DESARROLLO DE LA INVESTIGACIóN}

En esta sesión se describe una serie de actividades que en orden de ejecución fueron las siguientes: aplicación de un pretest para evaluar las habilidades dinámico-sistémicas en el grupo de estudiantes de la asignatura sistemas de producción, en el octavo semestre de ingeniería de petróleos de la UIs; las sesiones de trabajo que cubren desde una aproximación al pensamiento dinámico-sistémico, pasando por el uso de micromundos y terminando en una sesión de creación colaborativa de modelos de los sistemas de producción de pozos petroleros; aplicación del postest y valoración de resultados. 


\section{Pretest}

Se aplicó un test (ver Anexo 1) que fue diseñado y utilizado por Booth L. (2000) y replicado por Kapmeier F (2004), Ossimitz G (2002) y Armenia S. et al. (2004); existe otro experimento diferente, pero que en principio intenta develar las mismas habilidades realizado por Cronina M., et al. (2007). Los referentes antes citados fueron tenidos en cuenta para valorar los resultados del pretest. El principal objetivo del test consiste en hacer evidente la existencia o no de habilidades dinámico-sistémicas en los estudiantes, entendidas en este caso como la facilidad para la realización de la integración gráfica, al momento de abordar la solución de los dos desafíos; la bañera y el balance de pagos.

\section{Resultados de la aplicación del pretest}

Para tener un punto de referencia (ver Anexo 2) las soluciones al pretest se analizarán a la luz de los resultados obtenidos por los autores previamente citados, trabajos disponibles en línea. Al momento de hacer la interpretación de los datos es preciso indicar que en esta ocasión se tuvieron en cuenta la solución numérica y las habilidades cualitativas para la representación, medidas en términos de la tendencia de los niveles en función de los flujos.

Las siguientes tablas muestran los resultados obtenidos por los estudiantes y su comparación con trabajos previos.

Tabla 1. Resultados del desafío 1: la bañera

\begin{tabular}{|c|l|c|c|c|c|c|c|}
\hline \multirow{2}{*}{ NRO } & \multicolumn{1}{|c|}{ CRITERIO } & Pineda & Sterman & Ossimitz & Kapmeier & Kapmeier & Armenia \\
\cline { 2 - 8 } & UIS & MIT & $\begin{array}{c}\text { University of } \\
\text { Klagenfurt }\end{array}$ & SIMT & $\begin{array}{c}\text { Universität } \\
\text { Stuttgart }\end{array}$ & $\begin{array}{c}\text { Università } \\
\text { Tor Vergata }\end{array}$ \\
\cline { 2 - 8 } & Colombia & EEUU & Austria & Alemania & Alemania & Italia \\
\hline 2 & $\begin{array}{l}\text { Cuando el flujo de entrada excede el de } \\
\text { salida, el nivel crece. }\end{array}$ & 0,58 & 0,87 & 0,42 & 0,77 & 0,83 & 0,84 \\
\hline 3 & $\begin{array}{l}\text { Cuando el flujo de salida excede el de } \\
\text { entrada, el nivel decrece. }\end{array}$ & 0,50 & 0,86 & 0,43 & 0,68 & 0,82 & 0,80 \\
\hline 4 & $\begin{array}{l}\text { Los máximos y los mínimos del nivel } \\
\text { ocurren cuando el flujo neto es igual a cero } \\
\text { (t=4,8,12 y 16) }\end{array}$ & 0,46 & 0,89 & 0,56 & 0,64 & 0,82 & 0,92 \\
\hline 5 & $\begin{array}{l}\text { El nivel no muestra saltos discontinuos. } \\
\text { En cada instante de tiempo el flujo es } \\
\text { continuo por tanto el nivel crece o decrece } \\
\text { linealmente. }\end{array}$ & 0,42 & 0,96 & 0,64 & 0,82 & 0,86 & 0,78 \\
\hline 6 & $\begin{array}{l}\text { La pendiente del nivel durante cada } \\
\text { segmento es la tasa de cambio. (+/- 25 } \\
\text { unid/t) }\end{array}$ & 0,25 & 0,73 & 0,26 & 0,50 & 0,70 & 0,73 \\
\hline 7 & $\begin{array}{l}\text { La cantidad añadida o removida al nivel en } \\
\text { cada segmento será el área bajo la curva de } \\
\text { flujo neto. En 4 periodos sube a 200 o cae } \\
\text { a 100. }\end{array}$ & 0,13 & 0,68 & 0,27 & 0,55 & 0,65 & 0,38 \\
\hline
\end{tabular}


Tabla 2. Resultados del desafío 2: el balance de caja

\begin{tabular}{|c|c|c|c|c|c|c|c|}
\hline \multirow{3}{*}{ NRO } & \multirow{3}{*}{ CRITERIO } & Pineda & Sterman & Ossimitz & Kapmeier & Kapmeier & Armenia \\
\hline & & UIS & MIT & $\begin{array}{c}\text { University of } \\
\text { Klagenfurt }\end{array}$ & SIMT & $\begin{array}{c}\text { Universität } \\
\text { Stuttgart }\end{array}$ & $\begin{array}{l}\text { Università } \\
\text { Tor Vergata }\end{array}$ \\
\hline & & Colombia & EEUU & Austria & Alemania & Alemania & Italia \\
\hline $1 \mathrm{c}_{\mathrm{c}}^{\mathrm{C}}$ & $\begin{array}{l}\text { Cuando el flujo de entrada excede el de salida, } \\
\text { el nivel crece. }\end{array}$ & 0,25 & 0,48 & 0,25 & 0,27 & 0,52 & 0,57 \\
\hline $2 e_{c}^{2}$ & $\begin{array}{l}\text { Cuando el flujo de salida excede el de entrada, } \\
\text { el nivel decrece. }\end{array}$ & 0,25 & 0,48 & 0,23 & 0,32 & 0,49 & 0,57 \\
\hline \begin{tabular}{l|l} 
& $I$ \\
$c$ \\
1
\end{tabular} & $\begin{array}{l}\text { Los máximos y los mínimos del nivel ocurren } \\
\text { cuando el flujo neto es igual a cero }(\mathrm{t}=2,6, \\
10 \text { y 14) }\end{array}$ & 0,25 & 0,39 & 0,23 & 0,32 & 0,49 & 0,98 \\
\hline $4 \mathrm{~F}$ & El nivel no muestra saltos discontinuos. & 0,75 & 0,99 & 0,71 & 0,86 & 0,94 & 0,65 \\
\hline 5 & $\begin{array}{l}\text { La pendiente del nivel en cualquier tiempo es } \\
\text { la tasa neta por tanto. a) cuando el flujo neto } \\
\text { es positivo y decrece el nivel crece a una tasa } \\
\text { decreciente. } 0<t<2,8<t<10 \text {. b) cuando el flujo } \\
\text { neto es negativo y decreciente, el nivel decrece } \\
\text { a una tasa creciente } 2<t<4,10<t<12 \text {. c) } \\
\text { cuando el flujo neto es negativo y creciente, el } \\
\text { nivel decrece a una tasa decreciente } 4<t<6 \text {, } \\
12<t<14 \text {. d) cuando el flujo neto es positivo y } \\
\text { creciente, el nivel crece a una tasa creciente } \\
6<t<8,14<t<16 \text {. }\end{array}$ & 0,25 & 0,30 & 0,10 & 0,23 & 0,34 & 0,55 \\
\hline $6 \mid \begin{array}{ll}\mathrm{c} \\
\mathrm{t}\end{array}$ & $\begin{array}{l}\text { La pendiente del nivel cuando la tasa neta esta } \\
\text { en su máximo es } 50 \text { unidades por periodo de } \\
\text { tiempo. } T=0,8,16\end{array}$ & 0,38 & 0,52 & & 0,23 & 0,25 & 0,41 \\
\hline $7 \mid \begin{array}{l}\mathrm{e} \\
\mathrm{t}\end{array}$ & $\begin{array}{l}\text { La pendiente del nivel cuando la tasa neta esta } \\
\text { en su mínimo es - } 50 \text { unidades por periodo de } \\
\text { tiempo. } T=4,12\end{array}$ & 0,42 & 0,51 & & 0,14 & 0,26 & 0,43 \\
\hline 8 & $\begin{array}{l}\text { La cantidad adicionada o restada del nivel } \\
\text { durante cada segmento de } 2 \text { periodos es el área } \\
\text { encerrada por el flujo neto es }+/ \text { - } 50 \text {. Por tanto } \\
\text { habrá picos de } 150 \text { unidades y un mínimo de } \\
50 \text { unidades. }\end{array}$ & 0,00 & 0,41 & 0,14 & 0,32 & 0,41 & 0,41 \\
\hline & MEDIA & 0,32 & 0,51 & 0,28 & 0,34 & 0,46 & 0,57 \\
\hline
\end{tabular}

Los desafíos se aplicaron en un curso normal, donde una persona ajena a ellos les propone hacer el ejercicio; esta situación pudo haber influido en los resultados. Hecha la anterior aclaración se puede dar inicio a la revisión de las dos tablas anteriores que muestran la relación de desempeño de estudiantes de distintas universidades que resolvieron los desafíos de la bañera y el balance de caja. De esa información es evidente el hecho de que los estudiantes de petróleos de la UIS, que trabajaron los desafíos, se disputan el último lugar con los estudiantes de la University of Klagenfurt, de Austria, quienes en promedio tuvieron un mejor desempeño en el desafío de la bañera ( 0.42 vs 0.40$)$ en tanto que los estudiantes locales respondieron de mejor forma en desafío del balance de caja ( 0.32 vs 0.28 ); lo que términos generales daría un desempeño total del 0.36 vs 0.35 a favor de la uis.

De la información se puede concluir también que los estudiantes UIS obtienen el mejor desempeño, luego de la continuidad de la función, en el entendimiento adecuado de la relación entre flujo positivo e incremento del nivel. Esto puede significar que en términos cualitativos hubo una buena comprensión del fenómeno, aún cuando aparecieron problemas 
en el tratamiento matemático del mismo. Hay resultados que aparecen con una cierta incoherencia y son los concernientes a los ítems 1 y 2 en cada desafío. Para el primero el promedio fue del $54 \%$ de acierto en la solución, mientras que en el segundo fue del $25 \%$. Una posible explicación se encuentra en el hecho de que la información relacionada con el flujo de entrada en el primer desafío tiene forma de «escalón» mientras que en el segundo tiene la forma de «sierra».

\section{Prácticas de modelado con micropet y evolución 3.5}

Se propuso la realización de un trabajo práctico con el micromundo y el software de modelado dividido en seis sesiones de dos horas cada una. Se trabajaron dos sesiones con el micromundo en las cuales los estudiantes se acercaron a la experimentación con modelos didácticos y escenarios de simulación predefinidos. Dos sesiones más fueron necesarias para un primer acercamiento a la dinámica de sistemas y las dos últimas sesiones de modelado colaborativo fueron planteadas y ejecutadas para la construcción de modelos de fenómenos como la «ley de Darcy»y «las curvas de declinación».

\section{Primera sesión}

Se programó trabajar un primer acercamiento al pensamiento sistémico. Seguidamente se trabajó con Micropet buscando que los estudiantes exploraran los diferentes lenguajes de la dinámica de sistemas al acudir al concurso del modelo didáctico del CDT (inversión segura y rentable, con períodos de tiempo y tasas de interés previamente definidas).

Los estudiantes realizaron los recorridos pertinentes pasando por cada uno de los ambientes del micromundo, mostrando particular interés por la forma en que se presentaban los resultados mediante gráficas. A medida que se avanzó en la práctica pudieron observar cómo al hacer experimentos variando parámetros y niveles, hacían conciencia de que sus propios modelos mentales anticipaban comportamientos errados; lo que los obligaba a hacer revisiones a la luz de los resultados. Lo importante era que mediante el modelo podían dar cuenta de cuál era la creencia causante de la equivocación.

\section{Segunda sesión}

En la segunda sesión se hizo un acercamiento a los fundamentos de la dinámica de sistemas. La primera acción realizada consistió en describir cada uno de los lenguajes de la dinámica de sistemas, desde el lenguaje en prosa, hasta el lenguaje de los gráficos. En esta primera parte no se observó mucho interés por lo que se optó por construir un modelo e ir recordando durante el proceso cada uno de los lenguajes; de esta manera los estudiantes se ocupaban en el computador siguiendo las instrucciones.

Los modelos construidos fueron aquellos que constituían los desafíos que semanas antes ellos habían resuelto en clase. La solución de los mismos los hacía caer en la cuenta de los errores cometidos en aquella ocasión. El primer modelo se resolvió usando lápiz y papel, 
luego se utilizó una hoja electrónica. Ir del papel y el lápiz a la hoja electrónica no solo les facilitó las operaciones matemáticas sino que en este caso el estudiante era consciente acerca de qué era lo que estaba haciendo.

Posteriormente se trabajó con evolución para resolver los mismos modelos, pero llamando la atención en lo referente a la relación de los íconos (nivel, flujo, parámetros, etc.) con las nociones de cambio y acumulación. Finalmente se recrearon modelos de la física como el movimiento uniformemente acelerado que tuvo dos efectos importantes, el primero es que los estudiantes de antemano conocían el fenómeno, por un curso previo de mecánica, esto los motivó para ver cómo se podía simular ese fenómeno; y lo segundo fue que lograron encontrar la relación entre la noción de derivada, la noción de cambio y la idea de flujo y también reconocieron al nivel como la integral o la relación de acumulación. Seguidamente se propuso modelar el circuito RLC, se llegó hasta el planteamiento del diagrama de flujos y niveles quedando como tarea la simulación del mismo en casa.

De esta segunda sesión se puede concluir que efectivamente partiendo de la simulación manual, aparentemente se obtienen mejores resultados cuando de aprender a modelar se trata.

\section{Tercera sesión}

Para la tercera sesión se llevó a cabo un trabajo de modelado colaborativo que partía de los conocimientos teóricos de la ley de Darcy y las curvas de declinación. De la práctica resultó un modelo que integraba los dos conocimientos, es decir, se logró integrar dos temas que en el curso se ven por separado. La ley de Darcy que es utilizada para dar cuenta de la disminución de presión en el yacimiento y las curvas de declinación que sirven para explicar la producción de petróleo a medida que avanza el tiempo. Luego de plantear la ley de Darcy se construyó un primer modelo tratando de replicarla. El resultado atrajo la atención de los asistentes al seminario pues la aproximación del modelo, al menos en términos cualitativos, fue bastante similar a lo esperado por ellos.

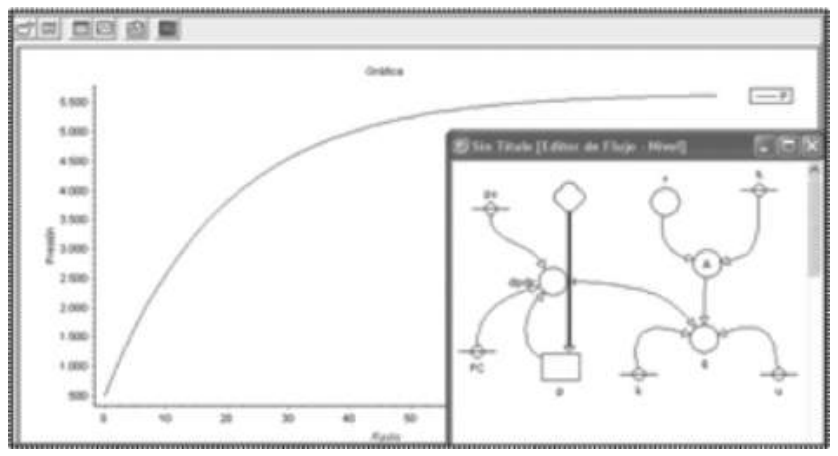

figura 4. Modelo de la ley de Darcy 
Luego de ese primer acercamiento se mostró cómo era posible no solo crear la representación del fenómeno mediante modelado y simulación, sino también replicar la solución analítica con la cual ellos habitualmente trabajan.

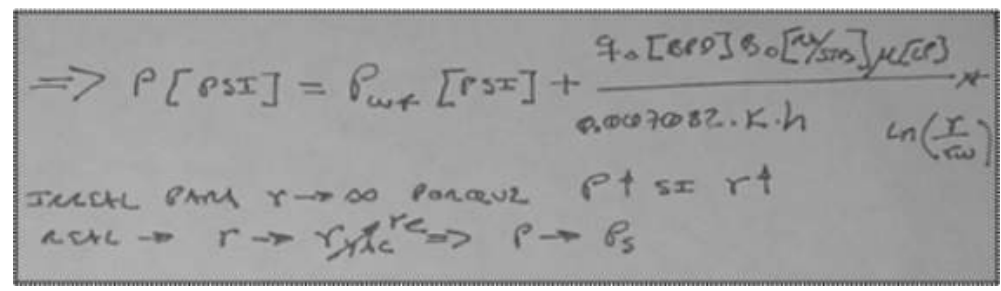

figura 5. Solución analítica

Apartir de la solución de la gráfica se puede encontrar la forma como cambia la presión respecto de la distancia del wellbore (pozo) a las fronteras del yacimiento.

Se obtuvo un modelo que atrajo la atención en la medida en que, según los estudiantes, podía contribuir para resolver los ejercicios sin tener que hacer operaciones matemáticas; además, percibían que al ser la presión una variable dependiente, su comportamiento dependerá de las demás variables, claro está, sin perder de vista que algunas de ellas no se pueden variar en la realidad, por ser propiedades del yacimiento.

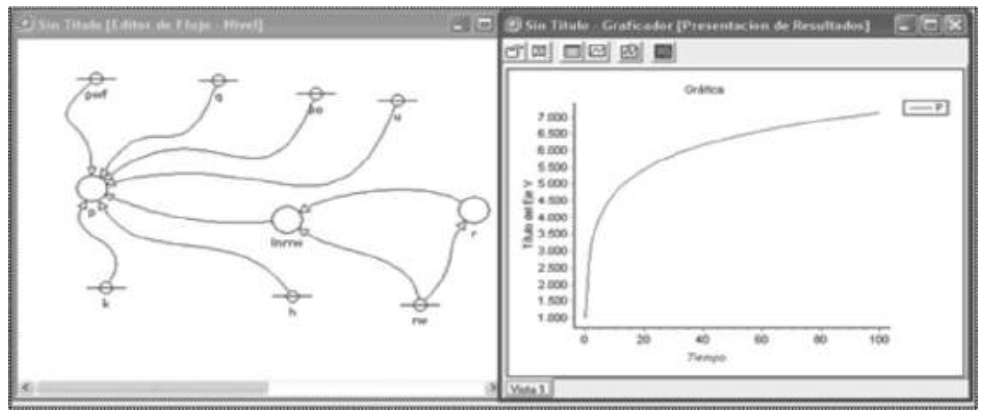

figura 6. Modelo y simulación de la presión vs radio

Fue evidente en este caso que se usan los elementos de la dinámica de sistemas, pero es absolutamente claro que eso no es modelado con dinámica de sistemas. Como se muestra en la Figura 6 existe una restricción relacionada con el hecho de que los yacimientos tienen límites, cosa que no se especifica en la solución analítica. Cuando se modela el fenómeno esta situación es posible resolverla.

Si se observa la Figura 7, en ella se incluye, como un elemento del sistema, la presión estática (PS), que es la que finalmente garantiza una solución mucho más cercana a la realidad. En el siguiente modelo se intentó recrear la forma en que se comporta la cantidad producida de petróleo durante un cierto tiempo, y que en el ámbito de la producción de petróleos se 
conoce con el nombre de curva de declinación. Luego de la discusión inicial se sugirió, por parte de los estudiantes, que el caudal, es decir, la forma en que cambia la cantidad de petróleo producida, debería ser expresado como consecuencia de la variación de la presión a través del tiempo y no en función del radio de drenaje. Para lograr esto se «volteó» la gráfica mediante un artificio matemático y se continuó el modelado según la expresión $\mathrm{Q}=\mathrm{dq} / \mathrm{dt}$, que es la expresión que da origen a la solución de la curva de declinación. El resultado fue el siguiente:

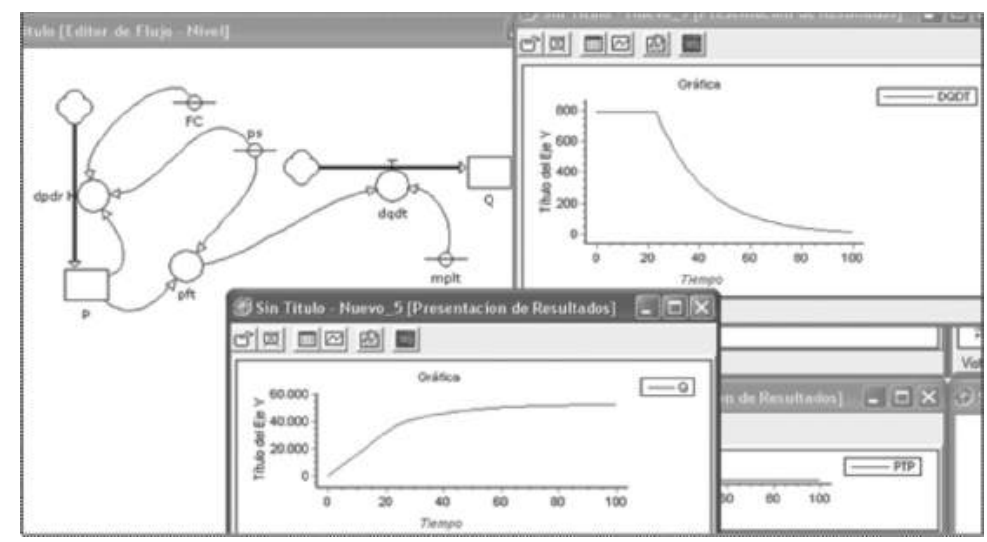

figura 7. La curva de declinación

La importancia de haber construido este modelo radicó en el hecho de que los estudiantes lograron entender que era posible dar explicaciones del fenómeno al asumirlo como si este fuera un sistema y no de la forma aislada en que se estudia el proceso de producción de pozos petroleros.

\section{Postest}

El test (ver Anexo 3) final buscó medir si había una diferencia significativa entre los estudiantes que tomaron el seminario y los que no, y entre los resultados obtenidos por otras universidades. De nuevo se acude a un ejercicio propuesto por Sterman J. (2002) y modificado por los autores para ubicarlo en el contexto de la asignatura, pero sin alterar los valores numéricos para efectos de medición y comparación.

\section{Resultados del postest}

Se presentaron al postest 37 de 40 estudiantes que conforman el grupo. Para realizar este análisis inicialmente se compararán los resultados obtenidos por aquellos que asistieron al seminario vs aquellos que no y se finalizará revisando los resultados a la luz de los obtenidos por estudiantes de otras instituciones (ver Anexo 4). 
Tabla 3. Resumen de resultados del postest

\begin{tabular}{|c|c|c|c|c|c|c|c|c|c|c|c|c|}
\hline \multirow{2}{*}{ CRITERIO } & \multicolumn{4}{|c|}{ CURSO COMPLETO } & \multicolumn{4}{|c|}{ CON SEMINARIO } & \multicolumn{4}{|c|}{ SIN SEMINARIO } \\
\hline & P1 & P2 & $\mathrm{P} 3$ & P4 & P1 & $\mathrm{P} 2$ & $\mathrm{P} 3$ & P4 & P1 & P2 & $\mathrm{P} 3$ & P4 \\
\hline Respuestas Acertadas & 18 & 13 & 0 & 0 & 11 & 9 & 0 & 0 & 7 & 4 & 0 & 0 \\
\hline Respuestas Equivocadas & 14 & 12 & 31 & 30 & 4 & 5 & 17 & 17 & 10 & 7 & 14 & 13 \\
\hline No Respuesta & 1 & 3 & 2 & 3 & 1 & 1 & 1 & 1 & 0 & 2 & 1 & 2 \\
\hline No Se Puede Determinar & 4 & 9 & 4 & 4 & 2 & 3 & 0 & 0 & 2 & 6 & 4 & 4 \\
\hline Participantes & 37 & 37 & 37 & 37 & 18 & 18 & 18 & 18 & 19 & 19 & 19 & 19 \\
\hline Porcentaje de respuestas acertadas & 0,49 & 0,35 & 0,00 & 0,00 & 0,61 & $\mathbf{0 , 5 0}$ & 0,00 & 0,00 & 0,37 & 0,21 & 0,00 & 0,00 \\
\hline Porcentaje de respuestas equivocadas & 0,38 & 0,32 & 0,84 & 0,81 & 0,22 & 0,28 & 0,94 & 0,94 & 0,53 & 0,37 & 0,74 & 0,68 \\
\hline Porcentaje de no respuesta & 0,03 & 0,08 & 0,05 & 0,08 & 0,06 & 0,06 & 0,06 & 0,06 & 0,00 & 0,11 & 0,05 & 0,11 \\
\hline Porcentaje de no se puede determinar & 0,11 & 0,24 & 0,11 & 0,11 & 0,11 & 0,17 & 0,00 & 0,00 & 0,11 & 0,32 & 0,21 & 0,21 \\
\hline
\end{tabular}

De la tabla anterior se puede apreciar que tan solo el cuarenta y nueve por ciento (49\%) del grupo total logra dar respuesta correcta a la primera pregunta y el treinta y cinco por ciento $(35 \%)$ a la segunda. El treinta y ocho por ciento $(38 \%)$ no acierta en la respuesta de la primera pregunta pero curiosamente solo el treinta y dos por ciento $(32 \%)$ responden de manera equivocada la segunda pregunta, el ochenta y cuatro por ciento (84\%) y el ochenta y uno (81\%) responden erróneamente la tercera y cuarta pregunta.

Respecto a las preguntas que no se respondieron, el tres por ciento (3\%) no contestaron la primera, el ocho por ciento (8\%) la segunda, el cinco por ciento $(5 \%)$ la tercera y el ocho por ciento $(8 \%)$ la cuarta. Finalmente la cuarta pregunta, según los estudiantes no se podía responder con la información aportada, la primera el once por ciento (11\%), la segunda el veinticuatro $(24 \%)$, la tercera el once por ciento $(11 \%)$ y la cuarta y el once por ciento $11 \%$. El alto puntaje en este ítem de la segunda pregunta explica porqué razón hubo menos porcentaje de respuestas equivocadas.

De lo anterior queda que los estudiantes no resolvieron el cincuenta y uno (51\%) de la primera pregunta, el sesenta y cinco por ciento $(65 \%)$ la segunda y el cien por ciento $(100 \%)$ de las preguntas tres y cuatro.

\section{Comparación de los resultados del postest frente a otras instituciones}

La importancia que esto pueda tener radica, especialmente, en que se podrá hacer una comparación de las habilidades dinámico-sistémicas de los estudiantes de la uis, específicamente de ingeniería de petróleos, con respecto a estudiantes en otras latitudes. 
Tabla 4. Resumen de resultados UIS

\begin{tabular}{|l|r|r|r|r|}
\hline CRITERIO & \multicolumn{3}{|c|}{ UIS - 2007} \\
\hline Porcentaje de respuestas acertadas & 0,49 & 0,35 & 0,00 & 0,00 \\
\hline Porcentaje de respuestas equivocadas & 0,38 & 0,32 & 0,84 & 0,81 \\
\hline Porcentaje de no respuesta & 0,03 & 0,08 & 0,05 & 0,08 \\
\hline Porcentaje de no se puede determinar & 0,11 & 0,24 & 0,11 & 0,11 \\
\hline
\end{tabular}

Tabla 5. Resumen de resultados UIS vs otras instituciones

\begin{tabular}{|c|c|c|c|c|c|c|c|c|}
\hline CRITERIO & \multicolumn{4}{|c|}{ Universität Stuttgart 2003 - 04} & \multicolumn{4}{|c|}{ Universität Stuttgart 2002- 03} \\
\hline Porcentaje de respuestas acertadas & 0,97 & 0,97 & 0.49 & 0,36 & 0,97 & 1,00 & 0,44 & 0,26 \\
\hline Porcentaje de respuestas equivocadas & ND & ND & ND & ND & ND & ND & ND & ND \\
\hline Porcentaje de no respuesta & ND & ND & ND & ND & ND & ND & ND & ND \\
\hline Porcentaje de no se puede determinar & 0,00 & 0,00 & 0,13 & 0,23 & 0,00 & 0,00 & 0,26 & 0,38 \\
\hline CRITERIO & \multicolumn{4}{|c|}{ MIT } & \multicolumn{4}{|c|}{ Carnegie Mellon University } \\
\hline Porcentaje de respuestas acertadas & 0,94 & 0,94 & 0,42 & 0,30 & 0,93 & 0,93 & 0,41 & 0,33 \\
\hline Porcentaje de respuestas equivocadas & ND & ND & ND & ND & ND & ND & ND & ND \\
\hline Porcentaje de no respuesta & ND & ND & ND & ND & ND & ND & ND & ND \\
\hline Porcentaje de no se puede determinar & 0,00 & 0,00 & 0,17 & 0,28 & ND & ND & ND & ND \\
\hline CRITERIO & \multicolumn{4}{|c|}{ SMIT 2003} & \multicolumn{4}{|c|}{$\begin{array}{c}\text { Sloan School of Management } \\
\text { MIT - } 173\end{array}$} \\
\hline Porcentaje de respuestas acertadas & 1,00 & 0,95 & 0,42 & 0,26 & 0,96 & 0,94 & 0,44 & 0,31 \\
\hline Porcentaje de respuestas equivocadas & ND & ND & ND & ND & 0,01 & 0,01 & 0,01 & 0,01 \\
\hline Porcentaje de no respuesta & ND & ND & ND & ND & 0,00 & 0,01 & 0,01 & 0,02 \\
\hline Porcentaje de no se puede determinar & 0,00 & 0,00 & 0,21 & 0,21 & 0,00 & 0,00 & 0,17 & 0,25 \\
\hline CRITERIO & \multicolumn{4}{|c|}{ Carlisle Public Schools - 46} & \multicolumn{4}{|c|}{$\begin{array}{l}\text { Worcester Polytechnic } \\
\text { Institute - } 80\end{array}$} \\
\hline Porcentaje de respuestas acertadas & 0,95 & 0,95 & 0,13 & 0,10 & 0,98 & 0,98 & 0,31 & 0,28 \\
\hline Porcentaje de respuestas equivocadas & ND & ND & ND & ND & ND & ND & ND & ND \\
\hline Porcentaje de no respuesta & ND & ND & ND & ND & ND & ND & ND & ND \\
\hline Porcentaje de no se puede determinar & ND & ND & ND & ND & ND & ND & ND & ND \\
\hline
\end{tabular}


En la medida en que no se dispone de la totalidad de los datos solo se hará referencia a los resultados de los aciertos, es decir, la primera fila para efectos de comparación. Considerando como una totalidad todos los resultados y debido a que los valores obtenidos son muy similares se tomará el promedio aritmético de los resultados.

Para la pregunta uno, los estudiantes de las demás instituciones tuvieron un promedio del noventa y seis por ciento $(96 \%)$ de respuestas acertadas, que evidentemente contrastan con el cuarenta y nueve por ciento (49\%) de las respuestas acertadas del grupo UIS en total y aún del sesenta y uno por ciento (61\%) que obtuvo el grupo que hizo el seminario. La pregunta dos fue resuelta por cerca del noventa y seis por ciento (96\%) de todos los estudiantes extranjeros, en tanto que sólo el treinta y cinco (35\%) del grupo UIS lo hizo bien y el cincuenta por ciento $(50 \%)$ de quienes participaron del seminario. El treinta y siete $(37 \%)$ y el veinte ocho por ciento (28\%) de las preguntas 3 y 4 respectivamente fueron correctamente resueltas en comparación con el cero por ciento $(0 \%)$ de los estudiantes del curso uIs completo.

Los anteriores resultados pueden sugerir que hay una equivocada percepción de la razón de cambio y la acumulación en los estudiantes locales, en la medida en que sus respuestas así lo hacen pensar. Si esto es así cabría preguntarse qué está sucediendo con la manera en que los estudiantes están aprendiendo el cálculo diferencial e integral, pues sería de esperarse que tuvieran claros los conceptos previamente mencionados.

Tabla 6. Resumen de promedios internacionales

\begin{tabular}{|c|c|c|c|}
\hline \multicolumn{4}{|c|}{ PROMEDIO } \\
\hline P1 & P2 & P3 & P4 \\
\hline 0,96 & 0,96 & 0,37 & 0,28 \\
\hline
\end{tabular}

Otro análisis busca recoger las diferencias entre el primer test y el segundo. Esta comparación debe tomarse con las debidas precauciones en la medida en que eran test diferentes aunque buscaban medir las mismas habilidades; además el grupo de estudiantes que participó fue diferente.

El primer test mostró que los estudiantes locales tuvieron un desempeño igual al treinta y seis por ciento (36\%) en tanto que las instituciones extranjeras obtuvieron un desempeño del cincuenta y seis por ciento (56\%). Como se mostró en los párrafos anteriores el desempeño en el postest aparentemente empeoró, siendo ampliamente superado el grupo, pero al compararlo con el grupo que tomó el seminario, este record baja considerablemente, lo que se presupone efecto del seminario.

Ahora debe hacerse una comparación entre el desempeño de los estudiantes que asistieron al seminario y los que no. Los que asistieron tuvieron un desempeño del sesenta y uno por ciento $(61 \%)$ y cincuenta por ciento $(50 \%)$ de acierto en las preguntas 1 y 2 contra un desempeño de los que no asistieron del treinta y siete por ciento $(37 \%)$ y veinte uno por ciento $(21 \%)$, respectivamente. 
Si se revisan las respuestas equivocadas, la relación es del veinte y dos por ciento (22\%) frente a un cincuenta y tres por ciento (53\%) y del veinte y ocho (28\%) frente a treinta y siete por ciento (37\%) manteniéndose una diferencia bastante significativa a favor de los asistentes al seminario.

Si bien es cierto que la muestra no es muy grande, si llama la atención el hecho de que los estudiantes que asistieron al seminario hayan tenido un mejor desempeño comparado con los que no. Otra de las mediciones que se propuso realizar fue con respecto al desempeño del grupo piloto frente al grupo de control. De un análisis bastante simple y partiendo de los promedios de notas se encontró que el promedio del grupo total fue de 3,67 en tanto que el subgrupo que asistió al seminario tuvo un promedio de $\mathbf{3 , 8 7}$ y el que no uno de $\mathbf{3 , 5 2}$. Revisando las cifras el desempeño del grupo que asistió al seminario estuvo un diez por ciento (10\%) por encima del que no.

\section{Posibles explicaciones del resultado del postest}

Hay dos posibles explicaciones para que ninguno de los estudiantes pudiera responder correctamente las preguntas tres y cuatro. La primera es que hubo una evidente percepción equivocada de la noción de cambio y la noción de acumulación, al punto de llegar incluso a confundirlas. La segunda razón es que no hubo suficiente estímulo que garantizara una ejecución del cuestionario con un mejor esfuerzo pues debe tenerse en cuenta que estaban presentando un parcial, que tenía un valor del veinticinco por ciento (25\%) de la asignatura.

\section{CONCLUSIONES}

Los resultados obtenidos en los pretest y los postest indican que los estudiantes a quienes se les aplicaron los test muestran percepciones equivocadas de los conceptos de razón de cambio y acumulación, que son propias del cálculo diferencial e integral.

Un acercamiento al pensamiento dinámico sistémico, como el realizado por los estudiantes de sistemas de producción de pozos petroleros, mejora su habilidad para la comprensión de fenómenos físicos, como fue evidenciado por el postest y las notas finales del curso.

El uso de micromundos, con modelos de simulación dinámica contribuye para mejorar la percepción de los fenómenos físicos, como la variación de la presión en un pozo petrolero y la declinación de la producción.

La descripción de los procesos de aprendizaje de la dinámica de sistemas contribuye para mejorar las habilidades dinámico-sistémicas de los estudiantes en la medida que mejora la percepción de los niveles y los flujos.

Al trabajar con modelos de simulación es preciso no caer en la trampa del modelo, es decir, se debe estar consciente de que se trabaja con una representación de la realidad y, además, que este es restringido a la perspectiva del modelista. 
Cuando se parte de la simulación manual, en un proceso de modelado, se obtienen mejores resultados cuando se está en el proceso de aprender dinámica de sistemas; esto se constituye en un punto de partida para futuras investigaciones sobre el aprendizaje del proceso de modelado.

\section{REfERENCIAS BIBLIOGRÁfICAS}

ANDRADE, H., et al. Pensamiento sistémico: diversidad en búsqueda de unidad. Bucaramanga: Ediciones UIS. 2001.

ARMENIA, Stéfano y ORONI, Ricardo. Bathtub Dynamics At The “Tor Vergata” University In Rome Italy. En: Proceedings of the 22nd International System Dynamics Conference, Albany, NY. 2004.

BOOTH S. Linda y STERMAN John D. Bathtub Dynamics: Initial Results of a Systems Thinking Inventory, System Dynamics Review; Winter; 16, 4; ABI/INFORM Global. 2000 .

CRONINA, Matthew A. y GONZÁLEZ, Cleotilde. Understanding the building blocks of dynamic systems, System Dynamics Review, 23. (2007).

DE ZUBIRÍA, J. Los modelos pedagógicos. 2. edición. Bogotá: Aula Abierta Magisterio. 2006.

KAPMEIER, Florián. Findings from four years of Bathtub Dynamics at Higher Educational Institutions in Stuttgart. En: Proceedings of the 22nd International System Dynamics Conference, Albany, NY. 2004.

LIZCANO, A. y PINEDA, E. MicrAS: Micromundo para el estudio del ciclo de crecimiento económico de Adam Smith, un enfoque sistémico. Tesis de Grado en Ingeniería de Sistemas. Universidad Industrial de Santander. Bucaramanga. 2000.

MARCHISIO, S., et al. Experiencia con uso de simulaciones en la enseñanza de la física de los dispositivos electrónicos. Primer congreso virtual latinoamericano de educación a distancia. Fundación latinoamericana para la educación a distancia. 2004. <www.flead.org>

NIND, T. E. W. Fundamentos de producción y mantenimiento de pozos petroleros. México: Limusa. 1987.

OSSIMITZ, Günther. Stock-Flow-Thinking and Reading stock-flow-related Graphs: an empirical investigation in dynamic thinking abilities. En: Proceedings of the 20nd International System Dynamics Conference, Palermo, Italy. 2002.

PINEDA, E. La simulación dinámica como posible apoyo a la comprensión de los sistemas de producción de pozos petroleros. Tesis de Grado en Maestría en Informática, Universidad Industrial de Santander. Bucaramanga. 2007. 
RADZICKI, M., et al. Why every engineering student should study system dynamics. En 32nd ASEE/IEEE Frontiers in Education Conference. IEEE November 6 - 9, 2002, Boston, MA. 2002.

STERMAN, J. All models are wrong: reflections becoming a systems scientist. Syst. Dyn. Rev. 18. 2002.

ZORA, J. Hidrogeología. 2008. En: <http://www.eccentrix.com/members/hydrogeologie/ archhtmlesp/09100es.htm>. Consulta realizada el 13 de julio de 2008. 


\section{ANEXO 1. Pretest}

¿HA JUGADO EL JUEGO DE LA CERVEZA? SI__ NO_

\section{PRIMER DESAfÍO}

Considere el siguiente gráfico que representa una tina. En ella hay una llave que la llena a razón de una cierta cantidad de agua por unidad de tiempo y también hay un desagüe (ver el dibujo).

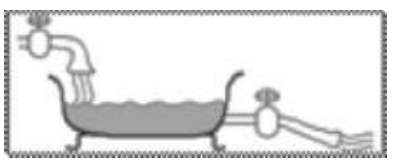

La siguiente gráfica muestra un posible comportamiento de las tasas de entrada y salida de agua de la tina. Con la información de la gráfica dibuje el comportamiento de la cantidad de agua en la tina, usando la gráfica que está en blanco. Suponga que inicialmente hay 100 litros en la tina.

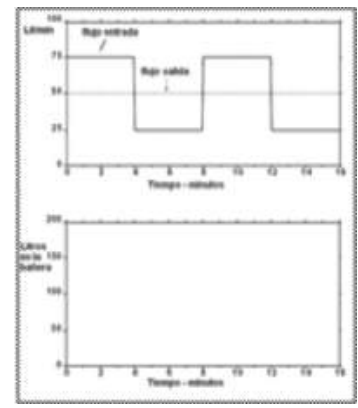

\section{SEGUNDO DESAfÍO}

Considere el balance de caja de una empresa. En ella se recibe dinero a una cierta tasa y se incurre en gastos a razón de otra tasa.

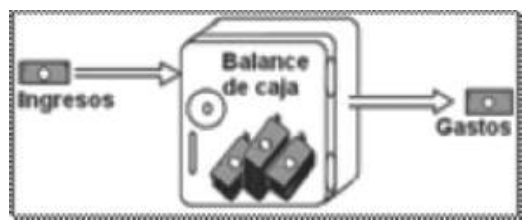

La siguiente gráfica muestra el comportamiento hipotético de los ingresos y gastos en caja. A partir de esta información dibuje el comportamiento del balance de caja utilizando la gráfica que está en blanco. 
Suponga que la cantidad inicial de dinero en la caja es de $\$ 100$.

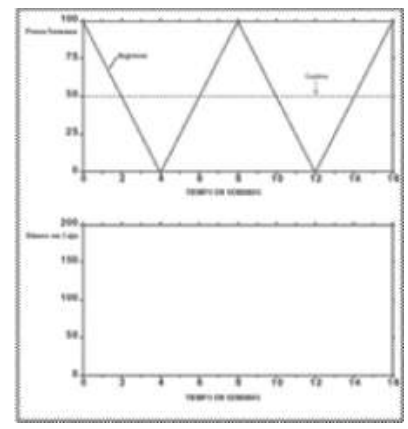

\section{TERCER DESAfíO}

Suponga que usted trabaja en una empresa de manufactura. La empresa mantiene un inventario de productos terminados y lo usa para atender las órdenes de los clientes. Históricamente, la empresa ha tenido órdenes en promedio de 10000 unidades por semana. Debido a que los pedidos de los clientes son absolutamente variables, la firma se esfuerza por mantener un inventario de 50000 unidades para proporcionarles un excelente servicio, esto es, poder cumplir al cien por ciento con cada orden, y ajustan la producción para cerrar cualquier brecha entre el inventario deseado y el actual. Aunque la empresa tiene una amplia capacidad para manejar variaciones en la demanda, toma un tiempo de cuatro semanas para ajustar el plan de fabricación y hacer el producto faltante.

Ahora imagínese que la tasa de la orden de los productos de la empresa se incrementa de manera repentina e inesperada en un $10 \%$, y permanece así de manera indefinida, según se indica en el gráfico siguiente. Antes del cambio en la demanda, la producción era igual a las órdenes de 10000 unidades/semana, y el inventario era igual al nivel deseado, es decir, 50000 unidades. Bosquejar la trayectoria probable de la producción y del inventario en el último de los gráficos. Proporcionar una escala apropiada para el gráfico del inventario.

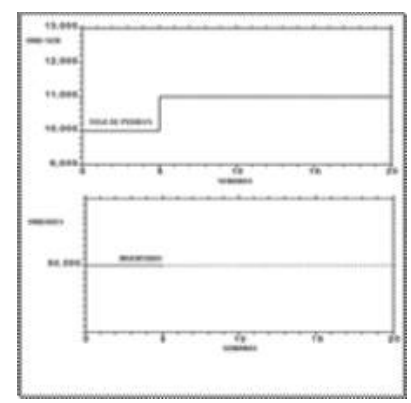

En caso de tener alguna duda consultar con el docente a cargo. 


\section{ANEXO 2. Solución al pretest}

Solución al desafío de la bañera.

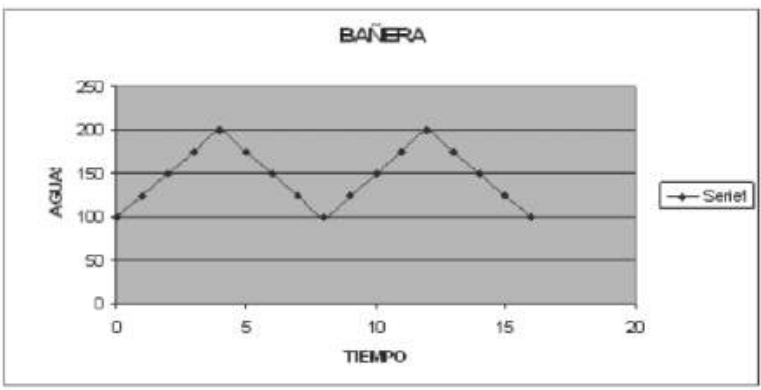

Solución al desafío del balance de caja.

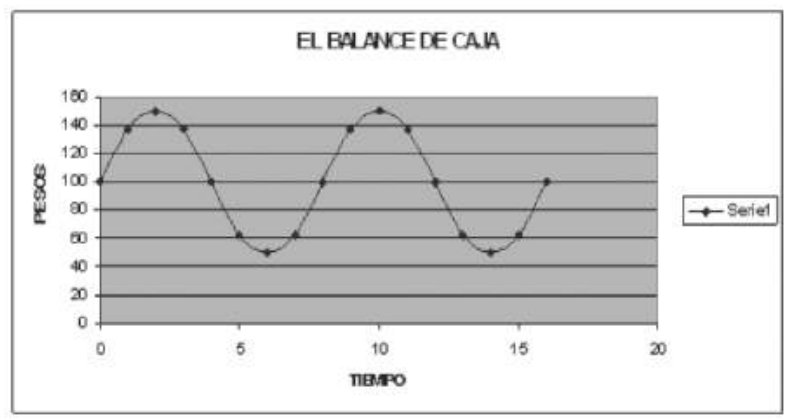

Solución al desafío de la producción en la empresa.

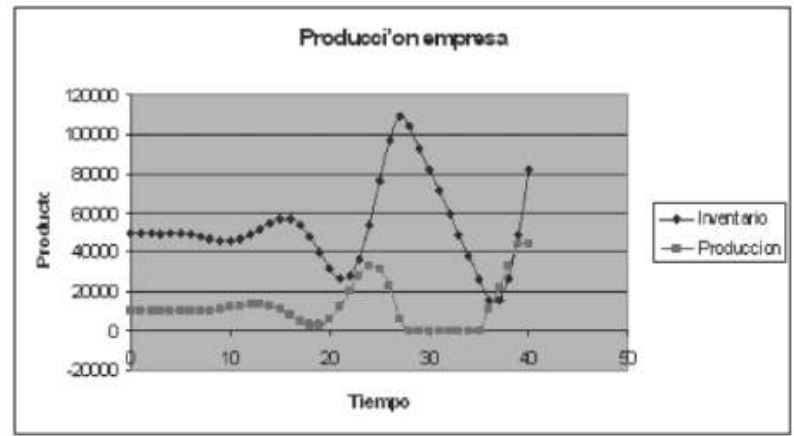




\section{ANEXO 3. Postest}

El siguiente gráfico muestra la cantidad de petróleo producida por un pozo, el cual se deposita en un tanque para luego ser procesado. La gráfica continua muestra cuánto petróleo entra al tanque mientras que la punteada se refiere al que sale; todo esto para un periodo de treinta días.

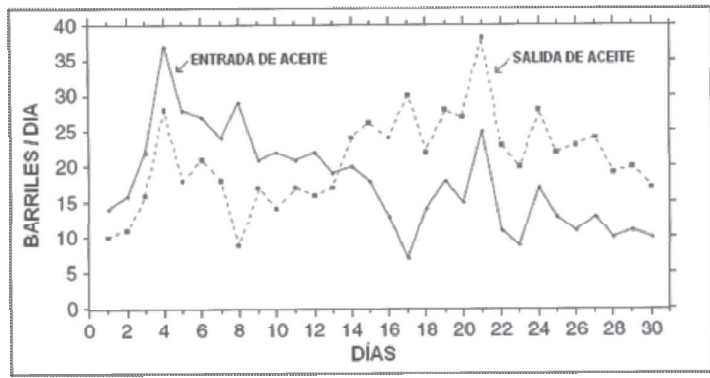

Chulee la caja $\checkmark$ si la respuesta no puede ser determinada por la información proveída.

1. ¿Durante cuál día se produjo más petróleo?

Día no se puede determinar

2. ¿Durante cuál día se procesó más petróleo?

Día no se puede determinar

3. ¿Durante cuál día hubo más petróleo en el tanque?

Día no se puede determinar

4. ¿Durante cuál día hubo menos petróleo en el tanque?

Día no se puede determinar 


\section{ANEXO 4. Solución al pretest}

1. ¿Durante cuál día se produjo más petróleo?

Día 4 no se puede determinar

2. ¿Durante cuál día se procesó más petróleo?

Día 21 ..no se puede determinar

3. ¿Durante cuál día hubo más petróleo en el tanque?

Día 13 ........................................no se puede determinar

4. ¿Durante cuál día hubo menos petróleo en el tanque?

Día 30 no se puede determinar 